INPLASY

PROTOCOL

To cite: Li et al. Comparative Efficacy and Safety of Exercise Training and Rehabilitation for Pulmonary Hypertension: A Systematic Review and Network Meta-Analysis. Inplasy protocol 2021100011. doi:

10.37766/inplasy2021.10.0011

Received: 04 October 2021

Published: 04 October 2021

Corresponding author: Qingyong He

limione0101@163.com

Author Affiliation:

China Academy of Chinese

Medical Sciences.

Support: Beijing-Tianjin-Hebei Basic Re.

Review Stage at time of this submission: Preliminary searches.

Conflicts of interest:

None declared.

\section{Comparative Efficacy and Safety of Exercise Training and Rehabilitation for Pulmonary Hypertension: A Systematic Review and Network Meta-Analysis}

Review question / Objective: The objective of this metaanalysis is to estimate the effectiveness of different exercise trainings on patients with pulmonary hypertension in single or double-blinded randomized controlled trials.

Condition being studied: Pulmonary hypertension (PH) (defines as a mean pulmonary artery pressure $\geq 25 \mathrm{mmHg}$ at rest on right-heart catheterization) comprises a complex group of conditions, characterized by increased right ventricular after load, which ultimately leads to right heart failure.

Information sources: Relevant articles are searched and selected from published data in electronic databases PubMed, Cochrane Library, MEDLINE, Embase, CNKI, Wanfang and VIP database (from inception until October 1, 2021)with the restriction on language: English and Chinese.

INPLASY registration number: This protocol was registered with the International Platform of Registered Systematic Review and Meta-Analysis Protocols (INPLASY) on 04 October 2021 and was last updated on 04 October 2021 (registration number INPLASY2021100011).

\section{INTRODUCTION}

Review question / Objective: The objective of this meta-analysis is to estimate the effectiveness of different exercise trainings on patients with pulmonary hypertension in single or double-blinded randomized controlled trials.

Condition being studied: Pulmonary hypertension (PH) (defines as a mean pulmonary artery pressure $\geq 25 \mathrm{mmHg}$ at rest on right-heart catheterization) 
comprises a complex group of conditions, characterized by increased right ventricular afterload, which ultimately leads to right heart failure.

\section{METHODS}

Participant or population: Adults with $\mathrm{PH}$ (men and women; age 18 years), independent of whether the subjects are stable on therapy ( change of treatment over the past 3 months) are included in this study.

Intervention: Interventions in the exercise group include aerobic, interval, strength, combined training (aerobic and strength), respiratory training, etc.

Comparator: Interventions for the control group include exercise methods, such as aerobic, interval, strength, combined training (aerobic and strength), respiratory training, or no exercise training.

\section{Study designs to be included: RCT.}

Eligibility criteria: Studies will be included if they conforming to the following criteria: (1)randomized controlled trail (RCT) as study design;(2)Adults with $\mathrm{PH}$ (men and women; age 18 years), independent of whether the subjects are stable on therapy (i.e., change of treatment over the past 3 months) are included in this study. (3) RCTs of exercise training in $\mathrm{PH}$ patients are included. In this study, exercise training include aerobic, interval, strength, combined training (aerobic and strength), respiratory training. The exclusion criteria are: (1) non-RCTs, studies with insufficient data, duplicated publications, conference reports, systematic reviews, or different studies from same sample origin; (2) participants restricted neonatal patients or pediatric;(3) crossover, $2 \times 2$ factorial, retrospective studies.

Information sources: Relevant articles are searched and selected from published data in electronic databases PubMed, Cochrane Library, MEDLINE, Embase, CNKI, Wanfang and VIP database (from inception until
October 1, 2021)with the restriction on language:English and Chinese.

Main outcome(s): 1. Change in WHO functional class; 2. Six-minute walk distance (6MWD).

Quality assessment / Risk of bias analysis: Two review authors independently assess the included studies for risks of bias using the Cochrane 'Risk of bias' assessment tool (Higgins 2011). We assess the following domains: 1.Random sequence generation. 2.Allocation concealment. 3.Blinding of participants and personnel. 4.Blinding of outcome assessment. 5.Incomplete outcome data. 6.Selective outcome reporting. 7.Other potential sources of bias.

Strategy of data synthesis: The network meta-analysis is performed using Stata (version 16.0, Stata SE), Review Manager (version 5.3, The Nordic Cochrane Centre, Copenhagen, Den-mark), and $\mathbf{R}$ software (version 4.0.3, $R$ foundation for statistical computing). The odds ratio (OR) with corresponding $95 \%$ confidence interval $(\mathrm{Cl})$ and mean difference(MD) with corresponding $95 \%$ confidence interval (Cl) are utilized to compare different exercises with respect to various clinical outcomes. Indirect comparisons are performed based on the Bayesian framework model using the "gemtc" package of the $R$ 3.5.3 software. OS is estimated using the hazard ratio (HR) with $95 \%$ confidence interval $(\mathrm{Cl})$. A 2-tailed $p<0.05$ is considered statistically significant. Based on the deviance information criterion (DIC) value, a fixed, or random-effects model is applied to the Bayesian framework model for data analysis. The convergence of the models is assessed with Brooks-Gelman-Rubin, trace, and density plots. A funnel plot is used to assess publication bias. Rank probabilities are calculated to determine the hierarchy of treatments. The "mtc.anohe" command in the "gemtc" package is used to evaluate global heterogeneity, which is documented with the variance parameter I2; a value $>50 \%$ is considered indicative of significant heterogeneity. The geometry of the 
treatment network is established using the R software. Inconsistency is assessed if any closed loop existed in the network. The stability of the results is obtained by sensitivity analyses with discarding each study sequentially. we use surface under the cumulative ranking (SUCRA) probability, which presents as a percentage the efficacy or safety of every intervention that is always best without uncertainty. The Node-splitting approach was used to assess the inconsistency.

Subgroup analysis: About half of the causes of PAH in the Western population are IPAH, HPAH, and drug-associated PAH, and connective tissue diseases which systemic sclerosis accounts for about $2 / 3$ of are the most common cause in APAH. And the causes of PAH in the Asian population are significantly different from the Western population. In the Asian population, Congenital heart disease is the most common cause of PAH, followed by IPAH and PAH associated with connective tissue disease in which systemic lupus erythematosus and Sjogren's syndrome are the most common cause. Therefore, in order to avoid the difference of treatment outcomes because of populations from different districts, patients were divided into groups depending on Asian or nonAsian populations to verify the internal consistency of the results of the clinical trial or explore the optimal benefit population.

Sensitivity analysis: Stratified metaanalysis is performed on the included literature according to the size of the sample size to investigate whether the conclusions have changed.

Country(ies) involved: China.

Keywords: Exercise training; Pulmonary hypertension; Exercise capacity;Quality of life; Randomized controlled trial.

Contributions of each author:

Author 1 - Min Li.

Author 2 - Anqi Li.

Author 3 - Yongen Yun.

Author 4 - Yixiang Li.
Author 5 - Xinyu Lu. Author 6 - Xin Zhao. 